\title{
Epicardial cyst eroding left ventricular wall mimicking as simple pericardial cyst: a case report
}

\author{
Dhiren shah ${ }^{1}$, Kishore Gupta ${ }^{1}$, Dhaval naik ${ }^{1}$, Amit chandan ${ }^{1}$, Chintan $\operatorname{sheth}^{1}$, and Gautam \\ $\operatorname{shah}^{1}$ \\ ${ }^{1}$ Care Institute of Medical Sciences
}

October 6, 2020

\begin{abstract}
Epicardial cysts are rarer benign tumors than pericardial cysts. 17- year old boy presented with chest pain and palpitations and was diagnosed as mediastinal mass (pericardial cyst) on the basis of MRI and echocardiography. Surgical resection via thoracotomy was planned. Intraoperatively, the cyst was found to be epicardial in origin with close proximity to posterior descending artery. Therefore resection was concluded via midline approach and cardiopulmonary support. Despite detailed preoperative investigation and assessment, misdiagnosis of cardiac masses cannot be completely ruled out. Any undesired intraoperative diagnosis should be carefully dealt and a safer alternative approach can be considered for a better outcome.
\end{abstract}

\section{Introduction}

Pericardial cysts are uncommon benign tumors, which account for $7 \%$ of all mediastinal tumors with incidence of 1:100,000 [1-3]. Epicardial cysts are much rarer than pericardial cysts, and their surgical management has seldom been reported. The surgical indications are when the cyst becomes symptomatic, infected or possibly malignant, grows progressively and compresses adjacent important structures $[2,4]$.

Mostly these cysts are asymptomatic and found incidentally on chest radiographs. $70 \%$ are located at the right cardiophrenic angle, $22 \%$ are at the left, and the rest are in the anterior or posterior mediastinum [5]. It is usually composed of a single layer of flat or cuboidal mesothelium containing clear, yellowish fluid [1, $6]$.

\section{Case report}

A 17-year old boy presented with chest pain and off \& on palpitations. Echocardiography revealed a large cyst $(7 \times 6.8 \times 7 \mathrm{~cm})$ around posteroinferior part of mediastinum and extending laterally. Magnetic resonance imaging (MRI) revealed well defined cystic mass lesion in the posterior, inferior and lateral aspect of left cardiac border appearing hyperintense on STIR sequences and hypotense on T1 sequence. (Figure 1)

Therefore, pericardial cyst removal via small thoracotomy was planned after taking infirmed consent. Thoracotomy was done but pericardium was found adherent posteriorly and superiorly and cyst appeared to be epicardial in origin. Therefore we planned to proceed via median sternotomy. Cyst was adhered to the pericardium and base was formed by visceral pericardium and myocardium of inferior wall of left ventricle. The cyst was most tightly attached to the left ventricle inferiorly. Thus, it was thought to have originated from the postero-inferior portion of LV. Cyst was found to be pushing the ventricles anteriorly. As the dissection of the mass was difficult and because of adhesions and close proximity to posterior descending artery we proceed with cardiopulmonary support.

Cyst wall was thick and vascular with feeder vessels over the surface. (Figure 2a) It was completely dissected and excised from its attachment. Inspection of the floor showed certain erosion of myocardial tissue, and 
the thickness of ventricular wall was attenuated. The cyst edges were trimmed, and multiple bleeding points along the cut edges were controlled with suture ligatures. The edges were then plicated over the attenuated ventricular wall with sutures to reinforce it. (Figure $2 b$ )

Histopathology revealed single layer of mesothelial cells. In addition to blood and lymphatic vessels, smooth muscle cells, lymphocyte infiltration, and fibrosis were observed (Figure 3).

The follow-up 2-dimensional echocardiography after 6 months did not show any abnormalities.

\section{Discussion}

We report our experience with a postero-inferior epicardial cyst which was successfully resected with onpump surgery. In our patient, excision was complicated because the cyst was located in close proximity to posterior descending artery. Resection of the cyst followed by reinforcement of the thinned ventricular wall could not have been accomplished without cardiopulmonary bypass support and the risk of ventricular wall injury leading to complicated course couldnot be completely ruled out. To circumvent such situation, we decided to operate on CPB. CPB may be required when cysts compress surrounding structures or erode the ventricular wall, coronaries $(2,3$, and 7$)$. We suggest that surgeon must be aware of possible need for CPB in such situations.

Preoperative differential diagnosis between a pericardial cyst and epicardial cyst is challenging because they cannot be differentiated on CT. (6) Although MRI is useful to differentiate cysts from other mediastinal lesions, it cannot differentiate between pericardial and epicardial cyst completely. This should broaden the spectrum of the potential appearance of differentials like epicardial masses intraoperatively. Role of cardiac magnetic resonance in tissue characterization to avoid erroneous preoperative diagnosis can have diagnostic importance. (9)

Coronary CT or angiography might be considered especially with regard to the degree of adhesions and location of the coronary vessels. However, it may not always exclude the possibility of involvement of coronaries because some literature reported epicardial cysts with involvement of the coronary vessels and CPB requirement to resect those cysts despite having negative findings $[1,2]$.

VATS/thoracotomy might be the first option but when invasion to important structures, including the left ventricle and coronary artery is suspected, median sternotomy with CPB should be considered. Off-pump resection might be indicated for uncomplicated cysts (8).

In our case, we were concerned about adhesions and close proximity of the cyst to surrounding structures thus the less invasiveness of thoracotomy was not considered beneficial enough to outweigh the risks associated with inadmissible complication.

Surgical resection on CPB can be a safe option when cyst lies in close proximity to important structures/erosion to ventricular wall. Detailed and meticulous preoperative investigation should be considered in large cyst in order to avoid any erroneous diagnosis.

\section{References}

1. Omeroglu SN, Omeroglu A, Ardal H, Erkilinc A, Bal E, Ipek G, et al. Epicardial mesothelial cyst located over the left anterior descending coronary artery.Tex Heart Inst J. 2004;31:313-5.

2. Hatemi, A. C., Kumbasar, U., Servet, E., Coskun, U., Bostan, C., \& Oz, B. (2012). Epicardial Cysts: Report of Two Rare Cases. The Heart Surgery Forum, 15(1), E49-E50. https://doi.org/10.1532/HSF98.20111117.

3. Scrofani R, Carro C, Beretta L, Antona C. Successful resection of an epicardial cyst. Ann Thorac Surg. 2002; 73:1633-4.

4. Kim JY, Koo HJ, Lee M, Kim GS, Jung SH. Epicardial cyst originating from right ventricle. Korean J Thorac Cardiovasc Surg 2013; 46:138-41.

5. Kutlay H, Yavuzer I, Han S, Cangir AK. Atypically located pericardial cysts. Ann Thorac Surg 2001; 72:2137-9. 
6. Ozasa A, Nakazawa K, Kamegai M, Miyake F, Murayama M, Sugai J. A case of epicardial cyst. Jpn Circ J. 1991; 55:53-7.

7. Chopra PS, Duke DJ, Pellett JR, Rahko PS. Pericardial cyst with partial erosion of the right ventricular wall. Ann Thorac Surg. 1991 May; 51(5):840-1. doi: 10.1016/0003-4975(91)90149-k. PMID: 2025099.

8. Kaneyuki, D., Sakata, T., Nomura, A. et al. Off-pump resection of a giant inflamed epicardial cyst. Surg case rep 4, 87 (2018). https://doi.org/10.1186/s40792-018-0495-3.

9. Zhou W, Srichai MB. Multi-modality Imaging Assessment of Pericardial Masses. Curr Cardiol Rep. 2017 Apr; 19(4):32. Doi: 10.1007/s11886-017-0845-y. PMID: 28315124.

Figure 1: MRI in transverse section and sagittal view showing well defined cystic mass in posterior and lateral portion abutting the left cardiac border.

Figure 2:

a) Intraoperative image depicting large size of cyst and feeder vessels over surface b) Post-operative image showing cyst floor with erosion of myocardial tissue and plicated margins.

Figure 3: Histopathological images showing a single layer of mesothelial cells and blood and lymphatic vessels, smooth muscle cells, lymphocyte infiltration, and fibrosis.
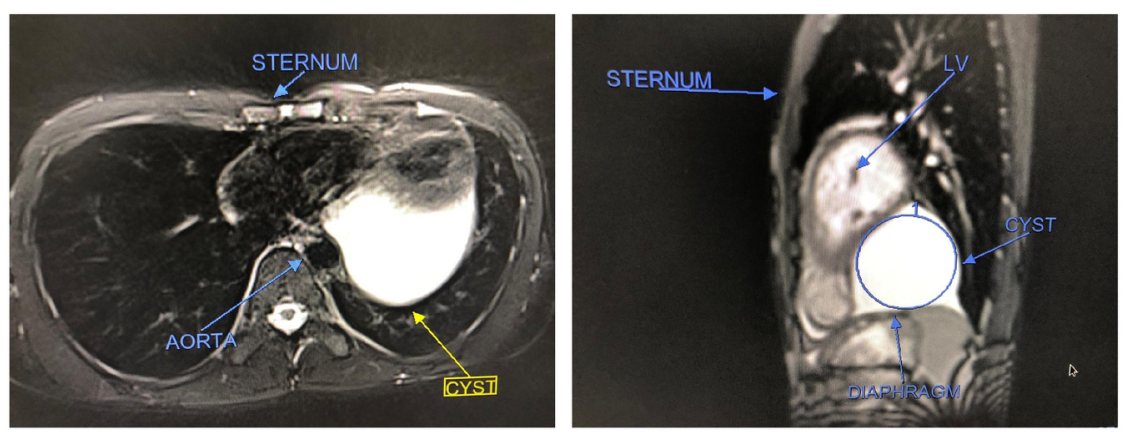

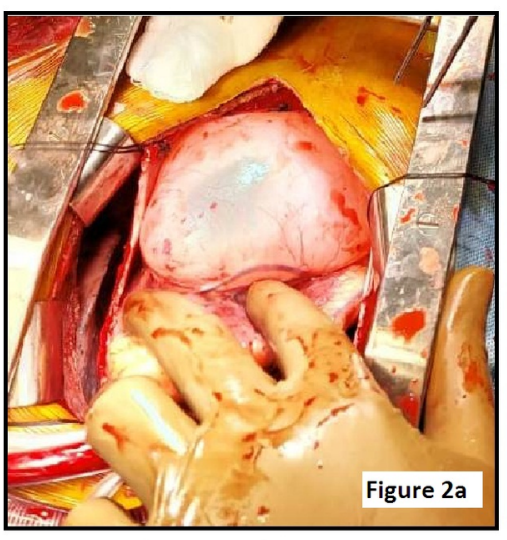

Pre resection

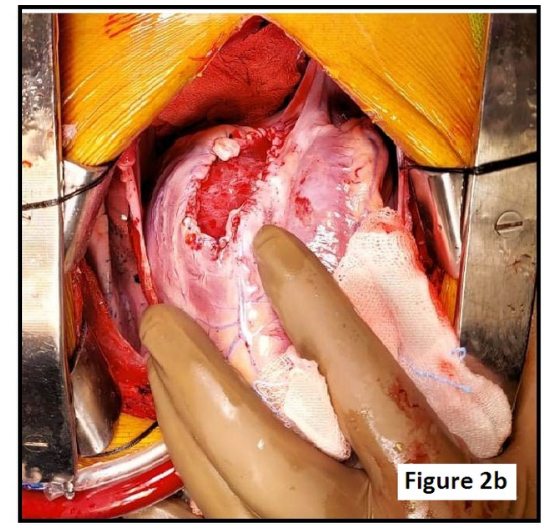

Post resection 

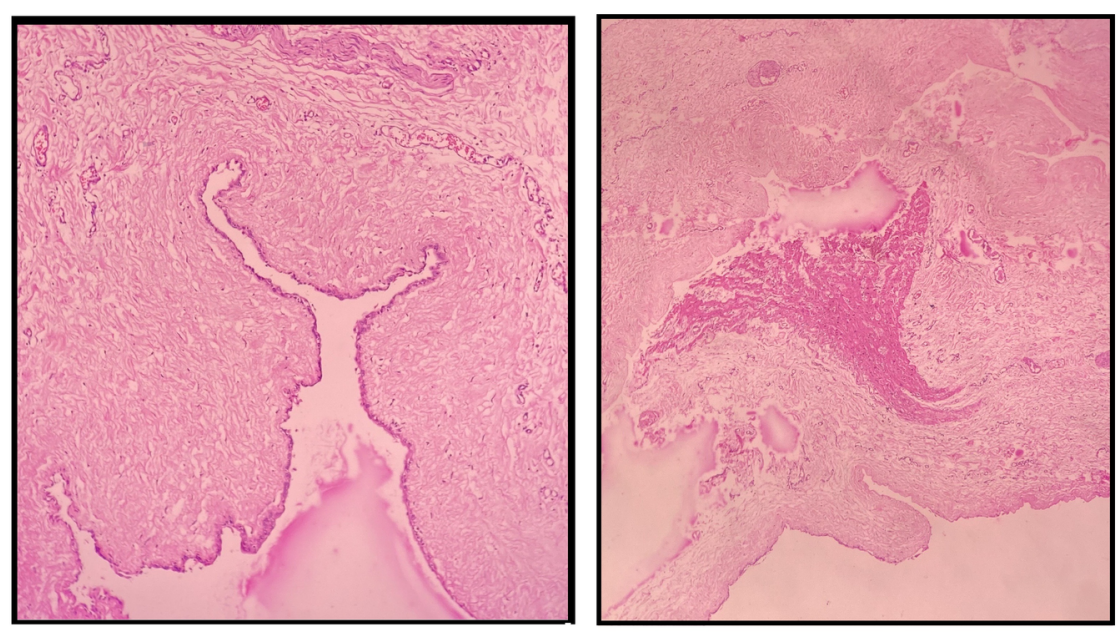